\title{
The Butterfly diagram leopard skin pattern
}

\author{
Maurizio Ternullo ${ }^{1}$ \\ ${ }^{1}$ INAF - Osservatorio Astrofisico di Catania, \\ v. S. Sofia 78, 95123 Catania, Italia \\ email: maurizio.ternullo@oact.inaf.it
}

\begin{abstract}
A time-latitude diagram where spotgroups are given proportional relevance to their area is presented. The diagram reveals that the spotted area distribution is higly dishomogeneous, most of it being concentrated in few, small portions ("knots") of the Butterfly Diagram; because of this structure, the BD may be properly described as a cluster of knots. The description, assuming that spots scatter around the "spot mean latitude" steadily drifting equatorward, is challenged. Indeed, spots cluster around at as many latitudes as knots; a knot may appear at either lower or higher latitudes than previous ones, in a seemingly random way; accordingly, the spot mean latitude abruptly drifts equatorward or even poleward at any knot activation, in spite of any smoothing procedure. Preliminary analyses suggest that the activity splits, in any hemisphere, into two or more distinct "activity waves", drifting equatorward at a rate higher than the spot zone as a whole.
\end{abstract}

Keywords. Sun: activity, sun: magnetic fields, sun: sunspots

\section{Introduction}

Throughout a century, the Butterfly Diagram (BD) bidimensional character has been described as the scattering of spots around a mean latitude, which has been assumed to steadily drift equatorward, through the center of the butterfly wings. As a consequence, most theoretical works are aimed at predicting the location and evolution of such a latitude. On the other hand, Ternullo $(2007 \mathrm{a}, \mathrm{b}, \mathrm{c})$ found, from the examination of cycles 20 to 22 , that the trace of the spot zone centroid results - in any hemisphere - from the quasi-biennial alternation of high-speed prograde phases with stationary or even retrograde phases, the average duration of the latter phases amounting to $\approx 35 \%$ of the cycle total duration. More recently, Ternullo (2008, 2010a,b) has shown that most of the spotted area is concentrated in small portions ("knots") of the BD, giving it a "leopard skin" aspect. Knots gather into two main streams per semicycle, drifting equatorward at a rate higher than the spot zone as a whole.

The present work is based on data sets compiled at the Royal Greenwich Observatory, integrated with data compiled by the US Air Force Solar Observing Optical Network and the National Oceanic and Atmospheric Administration. The valuable work of merging different archives was performed by Hathaway et al. (2003).

\section{Results}

For each one of the 1696 Carrington rotations from the 330th to the 2025th and for each one of the $841^{\circ}$-wide latitude strips in the interval $-42,+42^{\circ}$, the spotted area average values have been computed. The resulting figures are the elements of an $84 \times 1696$ array representing the quantitative counterpart of the "short line" diagram drawn by Maunder. This array has been smoothed by a triangular running window covering 5 Carrington rotations and visualized by means of level curves. A portion of the resulting diagram, 
concerned with cycles 15 to 17, is shown in Figure 1. A glance at this Figure reveals that the spotted area is distributed in butterfly wings with remarkable dishomogeneity: small, overspotted portions ( "knots") of the BD give it a "leopard skin" aspect. Knots are the signature of complexes of activity (Bumba \& Howard 1965; Gaizauskas et al. 1983) or sunspot nests (Castenmiller et al. 1986).

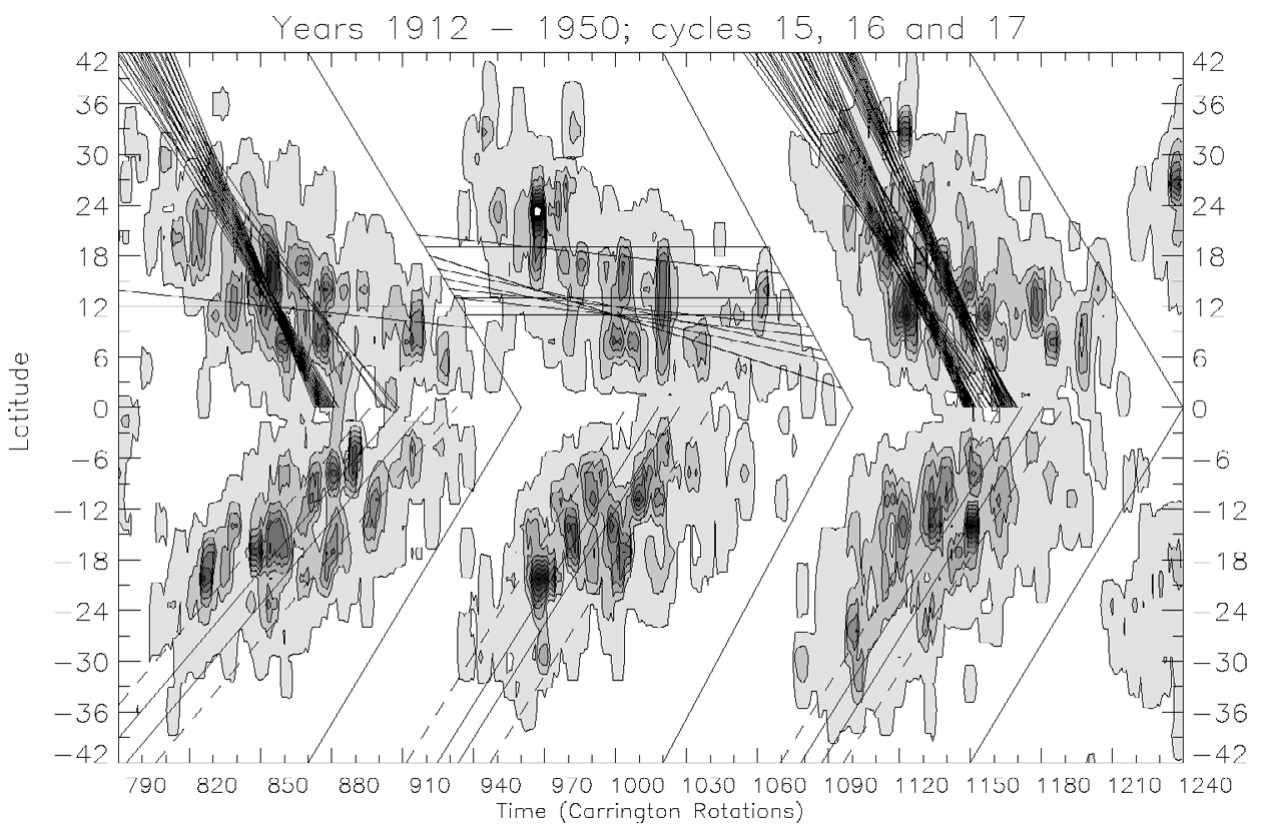

Figure 1. Butterfly Diagram for Carrington rotations 790-1300 (years 1912-1950). In any wing, the spotted area distribution is visualized by means of 10 level curves, dividing the range of spotted area values into equal intervals. The levels of grey qualitatively correspond to the spotted area density. The portion of the BD area lying between the two most external lines amounts to $\approx 70 \%$ of the butterfly total area, but hosts only $\approx 20 \%$ of the total spotted area. On the other hand, the 3rd level lines (circumscribing less than $\approx 15 \%$ of the butterfly total area) contain $\approx 50 \%$ of the total spotted area. Accordingly, the BD can be described as a cluster of small, highly concentrated spotted area aggregations (knots), distributed in a low-density spotted area population: this is the leopard skin pattern. In any southern-hemisphere wing, a couple of solid, oblique lines, 3 to $5^{\circ}$ from each other, marks the "depletion channel"; this is a band where the spotted area density is lower than in both adjacent comparison channels (dashed lines), both differences being significant at a level of significance not lower than $7 \sigma$; since many triplets of channels fulfil these statistical requirements, only the triplet associated with the most significant differences has been depicted for any wing. As regards the northern hemisphere wings, any triplet of channels fulfilling the same statistical requirements is schematically represented by the line passing through its center

Figure 1 shows that a knot may appear at latitudes either higher or lower than those of previous ones. Accordingly, because of the BD fragmentation into knots, and of their seemingly random occurrence, no continuous, steadily drifting equatorward line can represent the "mean latitude of spots": spots do not scatter on a line of this kind, but on as many latitudes as the knots.

Moreover, a glance at Figure 1 shows that, in cycles 15 s.h., 16 s.h. and 17 s.h., knots seem arranged into two oblique, discontinuous, roughly parallel streams, between which an underspotted band may be recognized. In order to get an objective definition 


\section{Cycle 16 s.h.} slope $(0 / y)$
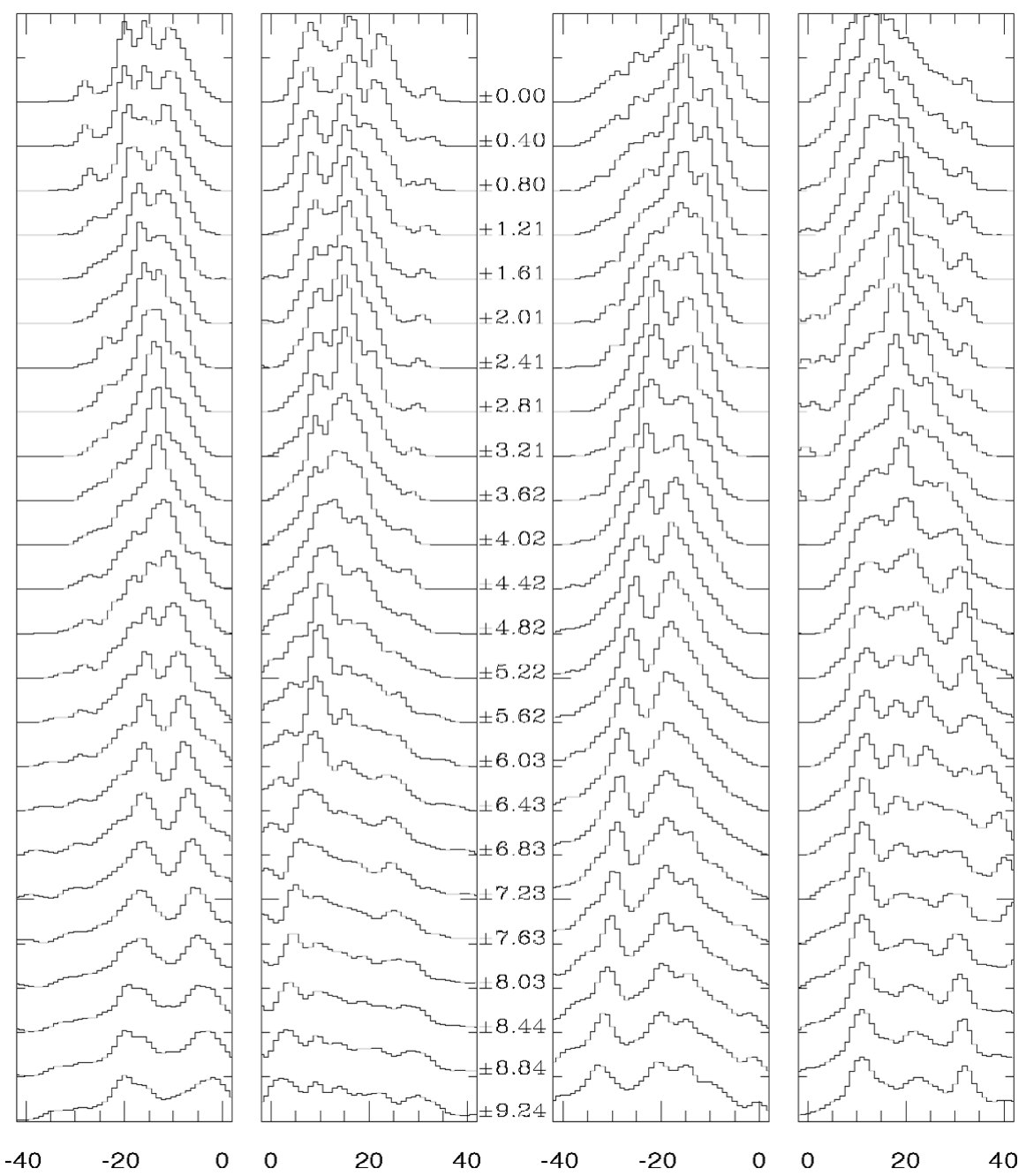

$20 \quad 40$

$-40$

00

$20 \quad 40$

Figure 2. Histograms showing the spotted area distribution in sets of parallel, oblique, $1^{\circ}$-wide elementary bands covering the cycle 15 and 16 butterfly diagram. The slope scans the range $\left[0, \pm 9.24^{\circ} \mathrm{y}^{-1}\right]$ (slopes are positive for the s.h. and negative for the n.h.). Sequentially examining histograms related to a given semicycle, it is easy to find that a small dip becomes a sharper and sharper depression until a special slope value is attained; for further slopes, the inverse process occurs.

of this band, I have adopted the procedure described in the following. I have virtually superimposed on any wing a set of $n$ parallel, oblique lines, $1^{\circ}$ from each other, so as to completely cover the wing surface with $n-1$ "elementary bands". Their slope is allowed to vary at small steps $\left(0.03^{\circ}\right.$ per Carrington rotation (CR), equivalent to $\left.0.402^{\circ} \mathrm{y}^{-1}\right)$, so as to finely scan the range $\left[0 ; 9.24^{\circ} \mathrm{y}^{-1}\right]$ in the s.h. and $\left[0 ;-9.24^{\circ} \mathrm{y}^{-1}\right]$ in the n.e. For any slope, the spotted areas, contained inside each elementary band, have been summed and the resulting sequence of sums (a sum for any elementary band) has been visualized by a histogram. Accordingly, a collection of histograms (a histogram for any slope) has been obtained for any wing (Figure 2). If an underspotted band actually crosses a wing with 
the unknown $\sigma^{\circ} \mathrm{y}^{-1}$ slope, we expect it to leave its signature in the histogram plotted for this slope value as a depression in its central portion. This approach has quantitatively confirmed that depletion channels actually exist in almost all the semicycles; that is, even in cases where - due to the knot pattern complexity - it was not trivial to single out them at a glance. Moreover, their slopes vary in a restricted range $\left(4 \approx 8^{\circ} \mathrm{y}^{-1}\right)$.

This novel picture of the spot cycle, as well as the finding that poleward phases are present in the spot zone drift (Ternullo, 2007a), enables us to give the following description of a typical spot cycle: two activity waves (or streams) form any spot cycle, in any hemisphere; the cycle begins with the activation of the first wave, at a latitude usually not larger than $24 \approx 30^{\circ}$; it generates the knots lying by the butterfly wing equatorward boundary; the second wave starts a couple of years after the first, at a latitude higher than the first one. Accordingly, spotgroups belonging to different waves lie in belts $\approx 6$ through $10^{\circ}$ apart. The depletion channel marks the separation between these two waves of activity; its slope is but the equatorward drift rate of each wave. The splitting of the spot cycle into two streams implies that, at any epoch, the spotted area latitudinal distribution is bimodal (Ternullo 1990). The sequence of activations and extinctions of knots belonging to either streams accounts for the zigzag displacements of the spot zone (Norton \& Gilman 2004), extensively described by Ternullo (1997, 2001, 2007a,b,c): indeed, the activation of the second wave, following by a couple of years the activation of the first one, mimics the first poleward drift of the spot zone centroid; afterwards, other retrograde phases occur because of the extinction of a low latitude knot followed by the activation of a high latitude one. According to the "standard picture", the spot zone centroid continuously drifts equatorward, at $\approx 2^{\circ} \mathrm{y}^{-1}$ average velocity (Waldmeier 1939; Hathaway et al. 2003); on the other hand, the afore-mentioned depletion-channel slope exceedes the "standard picture" rate by a factor $2 \approx 4$. Indeed, the former measures the drift rate of a stream, whilst the latter, deriving from traditional, unresolved pictures of the butterfly wings, involves the time interval between the first stream activation and the second extinction. The drift rate of any stream should be assumed as the spot zone actual drift rate. This new, higher value of the spot zone drift rate, as well as the activity splitting into two streams are features that any solar activity model should predict.

\section{Acknowledgements}

Ms. L. Santagati has reviewed the English form of this paper. This work was partially supported by Agenzia Spaziale Italiana (Accordo num. I/023/09/0 "Attività Scientifica per l'Analisi Dati Sole e Plasma - Fase E2" siglato fra INAF ed ASI)

\section{References}

Bumba, V. \& Howard, R., 1965, Astrophys. J., 141, 1502

Castenmiller, M. J.M. et al.,1986, Solar Phys., 105, 237

Gaizauskas, V. et al., 1983 Astrophys. J., 265, 1056

Hathaway, D. H., et al., 2003, Astrophys. J., 589, 665

Ternullo, M., 1990, Solar Phys., 127, 29

Ternullo, M., 1997 Solar Phys., 172, 37

Ternullo, M., 2001a Mem. S.A.It. 72, 565

Ternullo, M., 2001b Mem. S.A.It. 72, 681

Ternullo, M., 2007a Solar Phys., 240, 153

Ternullo, M., 2007b Mem. S.A.It., 78, 596

Ternullo, M., 2007c Astron. Nachr., 328, 1023

Ternullo, M., 2008 in Electronic Proceedings - 12th European Solar Physics Meeting, 8-12 September 2008 - Freiburg, Germany: http://espm.kis.uni-freiburg.de 
Ternullo, M., 2010a, Astrophys. Sp. Science, 328, 301; DOI: 10.1007/s10509-010-0270-9

Ternullo, M., 2010b, Mem. S.A.It. Suppl. 14, 202

Ternullo, M. 2010c, Advances in Plasma Astrophysics, Proceedings of the International Astronomical Union, IAU Symposium, Volume 274, 195

Waldmeier, M., 1939, Astron. Mitt Zürich, 14, 470 